\title{
Predictors Associated with a Prolonged Hospital Stay After Single-Port Extraperitoneal Robotic Radical Prostatectomy: A Comparative Analysis of Outpatient Versus Inpatient Care
}

\author{
Alireza Aminsharifi, MD, Clark A. Wilson, MD, Guilherme Sawczyn, MD, Soodong Kim, MD, \\ Louis Lenfant, MD, and Jihad Kaouk, MD
}

\begin{abstract}
Purpose: To compare the perioperative characteristics of patients receiving outpatient $v s$ inpatient care and to define predictors of inpatient care after single-port extraperitoneal robotic radical prostatectomy (RRP).

Patients and Methods: Data on 120 patients who underwent single-port extraperitoneal RRP were collected and categorized into two groups: Group I $(n=98)$ included patients who received outpatient care (i.e. discharged on postoperative day 0) and Group II $(n=22)$ comprised patients with inpatient care. Demographics and perioperative data were recorded and analyzed between the two groups. Multivariable binary logistic regression was used to determine factors associated with inpatient care.

Results: Most patients (98/120: 81.7\%) were discharged in few hours (median: 4.1 hours) after surgery (outpatient care: Group I $[n=98]$ ), whereas others (Group II $[n=22]$ ) received inpatient care (median hospital stay: 25.4 hours); $p<0.00001$. Most patients with inpatient care $(13 / 22,59.1 \%)$ were among the first initial 40 cases, whereas 37 out of last 40 patients received outpatient care $(p=0.005)$. Operative time was significantly shorter in patients with outpatient prostatectomy $(p=0.015)$. The amount of narcotics per patient (if administered) was also significantly greater in Group II $(p=0.006)$. With regression analysis, having medical comorbidities (odds ratio [OR]: $3.4195 \%$ confidence interval $[\mathrm{CI}]: 2.05-5.64 ; p=0.014$ ), a longer operative time (OR: $1.1595 \% \mathrm{CI}$ : $1.10-1.28, p=0.017$ ), as well as a higher dose of administered narcotics after operation (OR: $1.3195 \%$ CI: $1.08-1.61, p=0.005)$ were significant predictors of inpatient care after single-port extraperitoneal RRP.

Conclusion: A safe transition to outpatient care is feasible in patients undergoing single-port extraperitoneal RRP. In addition to associated medical comorbidities, a longer operative time and the amount of received narcotic after procedure were the most significant predictors of prolong hospital stay after single-port extraperitoneal RRP. These later modifiable predictors can be optimized with improvement of surgical techniques, intraoperative and postoperative pain management protocols through quality improvement initiatives.
\end{abstract}

Keywords: single-port radical prostatectomy, extraperitoneal prostatectomy, ambulatory surgery, robotic surgery, outpatient surgery, quality improvement

\section{Introduction}

T $N$ THE ERA of efficient value-based health care, initiatives to reduce health care costs while preserving quality patient care are highly rewarding. Minimally invasive surgery has been considered as one of the novel ways for improving the efficiency of health care by offering a shorter hospital stay and a smooth convalescence despite adoption of often ex- pensive and innovative technologies. ${ }^{1,2}$ This notion has been shown in robotic radical prostatectomy (RRP) literature, as a widespread standard of care for minimally invasive surgical management of localized prostate cancer. With comparable oncologic and functional outcomes, significant advantages in terms of postoperative recovery parameters have been shown in randomized trials. ${ }^{3,4}$ A Cochrane systematic review and meta-analysis of data also reported a significant shorter

Department of Urology, Glickman Urological and Kidney Institute, Cleveland Clinic, Cleveland, Ohio, USA.

The interim findings of this study were accepted as an oral abstract presentation at AUA2020 Washington, DC, USA. 
hospital stay with RRP compared with open technique (1.55 vs 3.27 days; $\Delta:-1.72,95 \% \mathrm{CI}:-2.19$ to -1.25$){ }^{5}$

In recent years, the safety and feasibility of outpatient robotic prostatectomy have been shown in selected patients. ${ }^{6-8}$ These studies have demonstrated that at least patients with minimal comorbidities or those who underwent a less complicated procedure (e.g., exclusion of lymph node dissection) can be considered as potential candidates for ambulatory robotic prostatectomy with an average hospital stay ranging between 12 and 24 hours. ${ }^{6-8}$ Cost-analysis data showed that same-day discharge can save $>\$ 2000$ per patient per day without affecting the surgical outcomes. ${ }^{9}$ Moreover, it has been recently shown that patients discharged on the same day after conventional robotic prostatectomy had higher quality of life indices than those who stayed $\geq 24$ hours. ${ }^{10}$

At our institution, with recent implementation of singleport robotic prostatectomy using da Vinci $\mathrm{SP}^{\circledR}$ system (Intuitive Surgical, Sunnyvale, CA), an increasing number of patients were discharged a few hours after their surgery without need for hospitalization or admission.

In this study, we present, analyze, and compare the perioperative characteristics of patients receiving outpatient vs inpatient care after single-port extraperitoneal RRP. To our knowledge, this is the first ever reported comparative trial to examine the predictors of inpatient care after single-port RRP; findings may be helpful to improve quality of care after robotic surgery.

\section{Patients and Methods}

\section{Patients}

Data of all patients who underwent single-port extraperitoneal RRP between October 2018 and December 2019 were prospectively collected and included in our Institutional Review Board-approved database. Patients were categorized into two groups according to their postoperative hospital stay: Group I included patients who received outpatient care (i.e., discharged on postoperative day 0) and Group II comprised patients with inpatient care (defined as staying $\geq 24$ hours after the surgery).

Preoperative assessment, staging, and risk stratification were according to standard guidelines. ${ }^{11}$ In general, all medical comorbidities (if present) were optimized before surgery with assistance from internal medicine/cardiology and anesthesiology services as needed. Patients were counseled preoperatively on the high likelihood of ambulatory surgery. They were discharged from the recovery room provided that their preoperative comorbidities (if present) remained stable and no perioperative medical (anesthesia) or surgical complications occurred.

\section{Operative setting}

Single-port extraperitoneal RRP with pelvic lymph node dissection was done for all patients. All surgeries were performed by one surgeon (J.K.). Details of surgical technique have been described previously. ${ }^{12,13}$ The extraperitoneal surgery was performed using with the patient positioned supine on a flat operative table. Intraoperative fluid management was optimized per anesthesiologist. Postoperative pain was controlled mainly by nonsteroidal analgesics with min- imal use of opioids. None of the patients had external drains placed after the surgery but all were discharged with a Foley catheter in place.

\section{Discharge criteria}

Postoperatively, patients considered fit for discharge demonstrated stable vital signs (heart rate $<100 /$ minutes, Systolic blood pressure $>100 \mathrm{~mm} \mathrm{Hg}$ ) and oxygen saturation $>90 \%$ with room air as well as postoperative hemoglobin level $\geq 10$ gr/dL and adequate urine output ( $\geq 30 \mathrm{~mL} / \mathrm{hour}$ ). In addition, patients at discharge were required to be ambulatory, tolerate oral diet, and have well-controlled pain without the need for intravenous narcotics. At the time of discharge, all patients were instructed to continue clear liquid diet with gradual advancement to regular diet, to take oral antibiotics until catheter is removed and to use stool softeners and oral analgesics as needed. Patients living more than a 2-hour drive away from the hospital were advised to stay near the hospital at least 1 day after discharge before travelling home. All patients had call access to the caregiver team in case of any questions or issues.

\section{Study outcomes and statistical analysis}

Demographic characteristics as well as intraoperative and postoperative data of patients in both groups were analyzed. Preoperative information included age, race, body mass index, Charlson comorbidity index, the presence of significant medical comorbidities (chronic obstructive pulmonary disease, chronic kidney disease, diabetes, hypertension, and coronary artery disease) and biopsy prostate cancer risk group. ${ }^{11}$

Intraoperative and postoperative variables included previous abdominal surgeries, operative time (calculated from skin incision to skin closure at the end of surgery), estimated blood loss, conversion rate, pain score at the time of discharge, length of hospital stay, and complications. The use of any additional port, change to a multiarm robotic, laparoscopic, or open approach was considered as conversion.

Perioperative complications were classified using the Clavien-Dindo classification system ${ }^{14}$; as major (grade $\geq$ III) or minor (grade $\leq \mathrm{II}$ ). Pain during hospital stay and at the time of discharge was scored using a numeric analog scale. ${ }^{15}$ The need for opioids for pain control after surgery as well as the amount of opioid after surgery (if administered) was recorded and analyzed between the two groups. All prostatectomy specimens were examined for final pathologic stage, surgical margins, lymph node status, and prostate size. For univariate analysis, Mann-Whitney $U$ test, Student's $t$-test (for continuous variables), and chi-squared (for categorical variables) were used, as appropriate. Binary logistic regression analysis was modeled for the assessment of significant risk factors associated with inpatient care after the surgery. All data were analyzed using SPSS ${ }^{\circledR}$ software v. 25 (IBM SPSS Statistics for Windows, Version 25.0. Armonk, NY: IBM Corp) and $p<0.05$ was considered significant.

\section{Results}

During the study period, 120 consecutive patients underwent single-port extraperitoneal RRP. There were no intraoperative anesthetic adverse event, intraoperative surgical complications, or conversion to an alternative procedure. No 
patient received blood transfusion during hospital course and most patients (98/120: 81.7\%) were discharged a few hours (median: 4.1 hours) after the surgery (outpatient care: Group I). Table 1 demonstrates demographic characteristics of patients with outpatient (Group I $n=98$ ) vs inpatient (Group II $n=22$ ) care after radical prostatectomy. As shown, tumor characteristics were similar between the two groups; however, a significant number of patients who required inpatient care after surgery (81.8\%) had at least one comorbidity (namely chronic obstructive pulmonary disease, chronic kidney disease, diabetes, coronary artery disease, or hypertension). In addition to possible concerns about comorbidities, other causes of inpatient care were unbearable postoperative pain, nausea and vomiting, and diet intolerance.

To evaluate the possible effect of learning curve on postoperative hospital stay we stratified and compared the rate of postoperative inpatient care between the initial and last 40 cases. Notably, most patients with inpatient care (13/22, $59.1 \%)$ were among the first initial 40 cases $(p=0.005)$.

Perioperative outcomes are compared in Table 2. The operative time was significantly shorter in patients with outpatient prostatectomy $(p=0.015)$. Pain scores at the time of discharge were similar in both groups; however, the rate of narcotic administration after surgery was significantly greater in patients with inpatient care $(59.1 \%$ vs $24.5 \%, p=0.001)$. Moreover, the amount of postprocedural narcotics per patient (if administered) was also significantly greater in the inpatient subgroup $(p=0.006)$ (Table 2). Postoperative complications as well as final prostatectomy histopathology and stage were comparable between the two groups (Table 2). In both groups, patients with pelvic lymphocele $(n=4)$ were managed with percutaneous drainage (Complication grade IIIIa).

Extraprostatic extension was detected in half of patients in this cohort (60/120 [50.0\%]). Overall, 23/29 (79.3\%) of patients with positive margin had high risk features (Extraprostatic extension, Gleason score 8-10, or positive nodes) on their final surgical pathology.
Among all significant perioperative variables in univariate analysis, with multivariable regression analysis, having medical comorbidities (odds ratio [OR]: 3.41 95\% CI: 2.055.64; $p=0.014)$, a longer operative time (OR: $1.1595 \% \mathrm{CI}$ : $1.10-1.28, p=0.017)$, as well as a higher dose of administered narcotics after the operation (in morphine milligram equivalent) (OR: 1.31 95\% CI: 1.08-1.61, $p=0.005$ ) were significant predictors of an increased odds of inpatient care after single-port extraperitoneal robotic prostatectomy (Table 3).

\section{Discussion}

Reducing postoperative morbidity, length of hospital stay, and its associated cost are strategies to improve the value of health care. With implementation of modern minimally invasive surgical technology, this strategy seems practical because several trials have shown a rapid recovery after minimally invasive surgery. ${ }^{1-5}$ Not only is outpatient care after robotic prostatectomy an interest for health care systems, a high rate of patient/family satisfaction $(87.5 \%-100 \%)$ has also been reported with this care pathway. ${ }^{7,16}$

The International Association for Ambulatory Surgery defines ambulatory surgery ( \pm extended recovery) as overall length of postoperative hospital stay $\leq 23$ hours. ${ }^{17}$ Using this definition, $>80 \%$ of patients in this cohort met the criteria of outpatient surgery (median hospital stay: 4.1 hours). Congnard et al. presented a large prospective cohort of planned outpatient robotic prostatectomy $(n=97){ }^{6}$ They developed and standardized a scoring system and discharge criteria; however, almost none of their patients met the discharge criteria on postoperative day 0 . They recognized nausea and vomiting as the most important barriers that interfere with same-day discharge protocol. ${ }^{6}$

In this study, we presented a model for outpatient prostatectomy by implementation of single-port extraperitoneal robotic prostatectomy program. With single-port surgery, we

Table 1. Characteristics of Patients Who Underwent Single-Port Extraperitoneal Robotic Radical Prostatectomy with Outpatient Versus InPatient Care

\begin{tabular}{|c|c|c|c|c|}
\hline & Total $(\mathrm{n}=120)$ & $\begin{array}{l}\text { Group I-outpatient } \\
\text { care }(\mathrm{n}=98)\end{array}$ & $\begin{array}{l}\text { Group II-inpatient } \\
\text { care }(\mathrm{n}=22)\end{array}$ & $\mathrm{p}$ \\
\hline Age, mean \pm SD (years) & $64.3 \pm 13.5$ & $64.0 \pm 13.7$ & $65.5 \pm 7.9$ & 0.31 \\
\hline Race, \%white & $107 / 120(89.2 \%)$ & $86 / 98(87.8 \%)$ & $21 / 22(95.4 \%)$ & 0.3 \\
\hline $\mathrm{BMI}\left(\mathrm{Kg} / \mathrm{m}^{2}\right)$, mean $\pm \mathrm{SD}$ & $29.3 \pm 17.4$ & $29.1 \pm 15.5$ & $30.3 \pm 16.4$ & 0.29 \\
\hline $\begin{array}{l}\text { Charlson comorbidity index, } \\
\text { median (IQR) }\end{array}$ & $4(4-5)$ & $4(3-5)$ & $4(4-5)$ & 0.38 \\
\hline Medical comorbidities ${ }^{\mathrm{a}}(\%)$ & $72 / 120(60.0 \%)$ & $54 / 98(55.1 \%)$ & $18 / 22(81.8 \%)$ & 0.02 \\
\hline Previous abdominal surgery (\%) & $35 / 120(29.2 \%)$ & $25 / 98(25.5 \%)$ & $10 / 22(45.5 \%)$ & 0.06 \\
\hline $\begin{array}{l}\text { Preoperative PSA (ng/mL), } \\
\text { median IQR }\end{array}$ & $6.1(6.0-9.0)$ & $6.1(4.8-9.0)$ & $6.6(4.9-9.3)$ & 0.095 \\
\hline \multicolumn{5}{|l|}{ Biopsy GrGp } \\
\hline GrGp1 & $15 / 120(12.5 \%)$ & $14 / 98(14.3 \%)$ & $1 / 22(4.5 \%)$ & \\
\hline GrGp2-3 & $80 / 120(66.7 \%)$ & $64 / 98(65.3 \%)$ & $16 / 22(72.7 \%)$ & 0.46 \\
\hline GrGp4-5 & $25 / 120(20.8 \%)$ & $20 / 98(20.4 \%)$ & $5 / 22(22.7 \%)$ & \\
\hline \multicolumn{5}{|l|}{ Prostate cancer risk group } \\
\hline Low risk & $13 / 120(10.8 \%)$ & $12 / 98(12.2 \%)$ & $1 / 22(4.5 \%)$ & \\
\hline Intermediate risk & $76 / 120(63.4 \%)$ & $61 / 98(62.2 \%)$ & $15 / 22(68.2 \%)$ & 0.58 \\
\hline High risk & $31 / 120(25.8 \%)$ & $25 / 98(25.6 \%)$ & $6 / 22(27.3 \%)$ & \\
\hline
\end{tabular}

${ }^{a}$ Including chronic obstructive pulmonary disease, chronic kidney disease, diabetes, hypertension, and coronary artery disease. $\mathrm{BMI}=$ body mass index; $\mathrm{GrGp}=$ grade group; $\mathrm{IQR}=$ interquartile range; $\mathrm{PSA}=$ prostate-specific antigen; $\mathrm{SD}=$ standard deviation . 
Table 2. Intra- and Perioperative Outcomes of Patients Who Underwent Single-Port Extraperitoneal Robotic Radical Prostatectomy with Outpatient Versus Inpatient Care

\begin{tabular}{|c|c|c|c|c|}
\hline & Total $(\mathrm{n}=120)$ & $\begin{array}{l}\text { Group I-outpatient } \\
\text { care }(\mathrm{n}=98)\end{array}$ & $\begin{array}{l}\text { Group II-inpatient } \\
\text { care }(\mathrm{n}=22)\end{array}$ & $\mathrm{p}$ \\
\hline Operative time (minutes), mean $\pm \mathrm{SD}$ & $203.2 \pm 60.6$ & $192.8 \pm 45.2$ & $229.5 \pm 46.5$ & 0.015 \\
\hline Estimated blood loss (mL), mean \pm SD & $197.2 \pm 53.8$ & $184.8 \pm 165.2$ & $252.3 \pm 172.7$ & 0.15 \\
\hline $\begin{array}{l}\text { Length of hospital stay (hours), } \\
\text { median (IQR) }\end{array}$ & $4.5(4.5-18.3)$ & $4.1(3.2-6.9)$ & $25.4(24.1-29.5)$ & $<0.00001$ \\
\hline Pain score at discharge, median (IQR) & $2(2-3)$ & $2(0-3)$ & $2(0.3-3.5)$ & 0.86 \\
\hline Need for opioids $(\%)$ & $37 / 120(30.8 \%)$ & $24 / 98(24.5 \%)$ & $13 / 22(59.1 \%)$ & 0.001 \\
\hline $\begin{array}{l}\text { MME/day in patients who used } \\
\text { opioids, mean } \pm \text { SD }\end{array}$ & $9.9 \pm 8.3$ & $8.3 \pm 6.4$ & $17.6 \pm 8.8$ & 0.006 \\
\hline $\begin{array}{l}\text { Prostate specimen weight }(\mathrm{g}), \\
\text { mean } \pm \text { SD }\end{array}$ & $57.2 \pm 27.6$ & $56.7 \pm 27.2$ & $59.4 \pm 29.9$ & 0.34 \\
\hline \multicolumn{5}{|l|}{ Pathologic GrGp } \\
\hline GrGp1 & $8 / 120(6.7 \%)$ & $7 / 98(7.1 \%)$ & $1 / 22(4.5 \%)$ & \\
\hline GrGp2-3 & $91 / 120(75.8 \%)$ & $73 / 98(74.5 \%)$ & $18 / 22(81.8 \%)$ & 0.76 \\
\hline GrGp4-5 & $21 / 120(17.5 \%)$ & $18 / 98(18.4 \%)$ & $3 / 22(13.7 \%)$ & \\
\hline \multicolumn{5}{|l|}{ Pathologic stage } \\
\hline $\mathrm{T} 2$ & $60 / 120(50.0 \%)$ & $52 / 98(53.1 \%)$ & $8 / 22(36.4 \%)$ & \\
\hline T3a,b & $60 / 120(50.0 \%)$ & $46 / 98(46.9 \%)$ & $14 / 22(63.6 \%)$ & 0.16 \\
\hline Positive lymph node & $6 / 120(4.7 \%)$ & $5 / 98(5.1 \%)$ & $1 / 22(4.5 \%)$ & 0.91 \\
\hline Positive surgical margin & $29 / 120(24.2 \%)$ & $\begin{array}{l}22 / 98(22.4 \%) \\
(18 / 22 \text { had high } \\
\text { risk features })\end{array}$ & $\begin{array}{l}7 / 22(31.8 \%) \\
(5 / 7 \text { had high } \\
\text { risk features })\end{array}$ & 0.35 \\
\hline Postoperative complications & $16 / 120(13.3 \%)$ & $13 / 98(13.3 \%)$ & $3 / 22(13.6 \%)$ & 0.96 \\
\hline Grade I-II & 9 & 7 & 2 & \\
\hline Grade III & 7 & 6 & 1 & \\
\hline 90-day undetectable PSA rate & $111 / 120(92.5 \%)$ & $90 / 98(91.8 \%)$ & $21 / 22(95.5 \%)$ & 0.34 \\
\hline 90 -day continence rate ( $0-1 \mathrm{pad} /$ day $)$ & $102 / 120(85.0 \%)$ & $83 / 98(84.7 \%)$ & $19 / 22(86.4 \%)$ & 0.84 \\
\hline
\end{tabular}

$\mathrm{MME}=$ morphine milligram equivalent.

were able to quickly transit from inpatient care (13 patients of the first 40 cases) to outpatient care (37 patients of last 40 cases). Such abrupt transition has also been previously reported in other centers with high-volume conventional robotic prostatectomy. ${ }^{9} \mathrm{We}$ also found that a longer operative time as well as the amount of narcotic medication received by the patient after his surgery were among the most significant predictors of a longer hospital stay (i.e., inpatient care). Both

Table 3. Results of Multivariable Binary Logistic Regression Analysis: Association Between Perioperative Variables and the Risk of InPatient Care After Single-Port

Extraperitoneal Robotic Radical Prostatectomy

\begin{tabular}{lcc}
\hline & \multicolumn{1}{c}{$\begin{array}{c}\text { Multivariable binary } \\
\text { logistic regression }\end{array}$} \\
\cline { 2 - 3 } Variable & $\begin{array}{c}\text { Odds ratio with } \\
\text { 95\% confidence } \\
\text { interval }\end{array}$ & $\mathrm{p}$ \\
\hline $\begin{array}{c}\text { Presence of medical } \\
\text { comorbidities }\end{array}$ & $3.41(2.05-5.64)$ & 0.014 \\
$\begin{array}{c}\text { Operative time } \\
\text { Administered narcotic } \\
\text { dose (MME/day) }\end{array}$ & $1.15(1.10-1.28)$ & 0.017 \\
\hline
\end{tabular}

${ }^{a}$ Including chronic obstructive pulmonary disease, chronic kidney disease, diabetes, hypertension, coronary artery disease. are potentially modifiable factors that can be further improved by quality improvement initiatives.

These findings are consistent with previous studies: using National Surgical Quality Improvement Program (NSQIP) database, through a propensity score matched model, Khalil et al. showed that patients with outpatient robotic prostatectomy tend to be younger and had a shorter mean operative time (187.8 vs 200.8 minutes, $p=0.008)$ compared with an inpatient surgery group. ${ }^{18}$

Abdominal surgery and operative time are significant predictors of intractable postoperative nausea and vomiting, which may lead to a longer hospital stay (OR: 2.19). ${ }^{6,19,20}$ Others have shown that every 30 -minute increase in operative time may be associated with a $60 \%$ increased risk of prolong postoperative nausea and vomiting (and, in turn, hospital stay). ${ }^{19,21}$ Consistent with the literature, our patients in Group II (inpatient care) had a longer mean operative time $(\Delta+36$ minutes) with an increased risk of longer hospital stay (OR: 1.15). As noted previously, many patients with an inpatient care pathway were in our first 40 cases; therefore, the difference in operative time may reflect the effect of learning curve.

Postoperative opioid administration is a well-known risk factor of intractable nausea vomiting and prolong recovery (OR: 1.93). ${ }^{19,22}$ As shown in this cohort study, a significant number $(\sim 60 \%)$ of those with inpatient care received postoperative opioids. Notwithstanding, on regression analysis, the amount of narcotics each patient received after surgery was associated with a higher risk of inpatient care (OR: 1.31). 
Consequently, the lesser (or "zero") amount of narcotic use, the shorter hospital stay. Unfortunately, because of a small sample size in patients with inpatient care $(n=22)$, this doseresponse assessment could not be completed by defining a possible cutoff value for the administration of narcotics that may not negatively affect the length of hospital stay. If future studies confirm these findings, to facilitate early discharge, development of an opioid-free anesthesia protocol ${ }^{23}$ for single-port extraperitoneal prostatectomy RRP can be proposed.

Postoperative narcotic administration is significantly associated with chronic opioid use ${ }^{24}$; therefore, this finding can also be rewarding with regard to the limitation of overall long-term narcotic use.

In contrast to some reports that recommend outpatient prostatectomy only in healthy patients with low-risk prostate cancer (i.e., selected cohorts), ${ }^{7,8}>50 \%$ of patients in our cohort had high risk features in their final pathology and we found noninferior short-term oncologic and functional outcomes with outpatient surgery protocol. Understandably, because of recent implementation of single-port robotic surgery, data need to mature to have a long-term assessment. We also showed that even patients with pelvic lymph node dissection can be safely considered for outpatient care pathway. A recent analysis of same-day surgery protocol for conventional robotic surgery $(n=246)$ also confirmed that at least at high-volume centers, pelvic lymph node dissection may not be a barrier for early discharge.

We do agree with others that a multidisciplinary care and communications between surgery, nursing, and anesthesia teams are crucial to ensure a safe discharge plan and to meet patient/family expectations. ${ }^{6-9}$

In this cohort study, we showed a safe transition to outpatient radical prostatectomy after implementation of single-port robotic surgery program. We found that along with associated medical comorbidities, longer operative time and the amount of administered narcotic after surgery were significant predictors of an inpatient hospital stay. However, the limitations of this study deserve to be mentioned. Importantly, because of retrospective nature of the study, we were not able to assess the causality and only the associations between the variables were examined. With a larger sample size (especially in patients who need inpatient care) and a longer follow-up, we could have defined a cutoff value for the use of narcotics with minimum (or no effect) on the length of stay.

Moreover, in this study we focused on postoperative narcotic use and we did not evaluate the anesthesia protocols and standards. We are aware that patients in this cohort were evaluated at a tertiary referral center and had their possible comorbidities controlled adequately before the surgery. Therefore, the result of this study should be interpreted with these assumptions. Patient satisfaction is an important index for evaluation of health care efficiency. We did not evaluate this parameter. However, it is likely that decreased hospital stay, less and smaller incisions, potentially less pain, and rapid recovery after single-port surgery may contribute overall patient satisfaction.

\section{Conclusion}

With increasing experience in single-port extraperitoneal robotic prostatectomy, a safe transition to outpatient care pro- tocol is feasible. Along with associated medical comorbidities, a longer operative time and the amount narcotics administered postoperatively were the most significant predictors of prolong hospital stay after single-port extraperitoneal robotic prostatectomy. These later modifiable risk factors can be improved with modifications in surgical techniques, as well as intraoperative and postoperative pain management protocols through quality improvement initiatives.

\section{Author Disclosure Statement}

J.K. has a consultant agreement with Intuitive Surgical.

\section{Funding Information}

No funding was received for this article.

\section{References}

1. Abrishami P, Boer A, Horstman K. How can we assess the value of complex medical innovations in practice? Expert Rev Pharmacoecon Outcomes Res 2015;15:369-371.

2. Abrishami P, Boer A, Horstman K. When the evidence basis breeds controversies: Exploring the value profile of robotic surgery beyond the early introduction phase. Med Care Res Rev 2019; DOI: 10.1177/1077558719832797.

3. Yaxley JW, Coughlin GD, Chambers SK, et al. Robotassisted laparoscopic prostatectomy versus open radical retropubic prostatectomy: Early outcomes from a randomised controlled phase 3 study. Lancet 2016;388:10571066.

4. Sooriakumaran P, Pini G, Nyberg T, et al. Erectile function and oncologic outcomes following open retropubic and robot-assisted radical prostatectomy: Results from the LAParoscopic prostatectomy robot open trial. Eur Urol 2018;73:618-627.

5. Ilic D, Evans SM, Allan CA, Jung JH, Murphy D, Frydenberg M. Laparoscopic and robotic-assisted versus open radical prostatectomy for the treatment of localised prostate cancer. Cochrane Database Syst Rev 2017;9:CD009625.

6. Congnard D, Vincendeau S, Lahjaouzi A, Neau AC, Chaize C, Estèbe JP, Mathieu R, Beloeil H. Outpatient robotassisted radical prostatectomy: A feasibility study. Urology 2019;128:16-22.

7. Berger AK, Chopra S, Desai MM, Aron M, Gill IS. Outpatient robotic radical prostatectomy: Matched-pair comparison with inpatient surgery. J Endourol 2016;30(Suppl. 1):S52-S56.

8. Banapour P, Elliott P, Jabaji R, Parekh A, Pathak A, Merchant M, Tamaddon K. Safety and feasibility of outpatient robot-assisted radical prostatectomy. J Robot Surg 2019;13:261-265.

9. Abaza R, Martinez O, Ferroni MC, Bsatee A, Gerhard RS. Same day discharge after robotic radical prostatectomy. J Urol 2019; DOI: 10.1097/JU.0000000000000353.

10. Bajpai RR, Razdan S, Barack J, Sanchez MA, Razdan S. Ambulatory robot-assisted laparoscopic prostatectomy: Is it ready for prime time? A quality of life analysis. J Endourol 2019 [Epub ahead of print]; DOI: 10.1089/end.2019.0261.

11. Mohler JL, Armstrong AJ, Bahnson RR, et al. Prostate cancer, version 1.2016. J Natl Compr Canc Netw 2016;14: 19-30.

12. Kaouk J, Bertolo R, Eltemamy M, Garisto J. Single-port robot-assisted radical prostatectomy: First clinical experience using the SP surgical system. Urology 2019;124:309. 
13. Bertolo R, Garisto J, Gettman M, Kaouk J. Novel system for robotic single-port surgery: Feasibility and state of the art in urology. Eur Urol Focus 2018;4:669-673.

14. Clavien PA, Barkun J, de Oliveira ML, et al. The ClavienDindo classification of surgical complications: Five-year experience. Ann Surg 2009;250:187-196.

15. Breivik H, Borchgrevink PC, Allen SM, Rosseland LA, Romundstad L, Hals EK, Kvarstein G, Stubhaug A. Assessment of pain. Br J Anaesth 2008;101:17-24.

16. Martin AD, Nunez RN, Andrews JR, Martin GL, Andrews PE, Castle EP. Outpatient prostatectomy: Too much too soon or just what the patient ordered. Urology 2010;75: 421-424.

17. https://www.iaas-med.com/index.php/iaas-recommendations/ extended-recovery-facilities (Accessed October 14, 2019).

18. Khalil MI, Bhandari NR, Payakachat N, Davis R, Raheem OA, Kamel MH. Perioperative mortality and morbidity of outpatient versus inpatient robot-assisted radical prostatectomy: A propensity matched analysis. Urol Oncol 2019 [Epub ahead of print]; DOI: 10.1016/j.urolonc.2019.07.008.

19. Öbrink E, Jildenstål P, Oddby E, Jakobsson JG. Postoperative nausea and vomiting: Update on predicting the probability and ways to minimize its occurrence, with focus on ambulatory surgery. Int J Surg 2015;15:100-106.

20. Odom-Forren J, Jalota L, Moser DK, Lennie TA, Hall LA, Holtman J, Hooper V, Apfel CC. Incidence and predictors of postdischarge nausea and vomiting in a 7-day population. J Clin Anesth 2013;25:551-559.

21. Sinclair DR, Chung F, Mezei G. Can postoperative nausea and vomiting be predicted? Anesthesiology 1999;91:109118.

22. Apfel CC, Philip BK, Cakmakkaya OS, et al. Who is at risk for postdischarge nausea and vomiting after ambulatory surgery? Anesthesiology 2012;117:475-486.
23. Blaudszun G, Lysakowski C, Elia N, Tramèr MR. Effect of perioperative systemic $\alpha 2$ agonists on postoperative morphine consumption and pain intensity: Systematic review and meta-analysis of randomized controlled trials. Anesthesiology 2012;116:1312-1322.

24. Hacker KE, Jung J, Graves JL, et al. Postoperative opioid prescribing in urology: Are we contributing to the national crisis? J Am Coll Surg 2018;227(Suppl. 2):e166.

Address correspondence to: Jihad Kaouk, MD

Department of Urology

Glickman Urology and Kidney Institute Cleveland Clinic

9500 Euclid Avenue, Q-10

Cleveland, $\mathrm{OH} 44195$

USA

E-mail: kaoukj@ccf.org

$\begin{aligned} & \quad \text { Abbreviations Used } \\ & \mathrm{BMI}=\text { body mass index } \\ & \mathrm{CI}=\text { confidence interval } \\ & \mathrm{GrGp}=\text { grade group } \\ & \mathrm{IQR}=\text { interquartile range } \\ & \mathrm{MME}=\text { morphine milligram equivalent } \\ & \mathrm{NSQIP}=\text { National Surgical Quality Improvement Program } \\ & \mathrm{OR}=\text { odds ratio } \\ & \mathrm{PSA}=\text { prostate-specific antigen } \\ & \mathrm{RRP}=\text { robotic radical prostatectomy } \\ & \mathrm{SD}=\text { standard deviation }\end{aligned}$

